\title{
Pediatric Dentistry during Coronavirus Disease-2019 Pandemic: A Paradigm Shift in Treatment Options
}

Amita Sharma ${ }^{1}$, Megha B Jain ${ }^{2}$

\begin{abstract}
Coronavirus disease-2019 (COVID-19) pandemic has affected the world in an aggressive manner and the healthcare fraternity has been at the forefront in this fight. Dental professionals are at a larger risk to get afflicted owing to the proximity to the oral cavity. Along with other verticals of dentistry, pediatric dentistry too has to adapt quickly keeping in mind the newer protocols so as to limit the increase of the global epidemic and the commencement of cross-infections. The purpose of this article is to present different treatment options for a pediatric dentist so as to achieve positive dental outcomes.

Keywords: Aerosol-free dentistry, Atraumatic restorative treatment, Caries, Chemomechanical caries removal, COVID-19, Pulpotomy, Silver diamine fluoride.

International Journal of Clinical Pediatric Dentistry (2020): 10.5005/jp-journals-10005-1809
\end{abstract}

\section{INTRODUCTION}

Coronavirus disease-2019 (COVID-19) has affected the world in an unprecedented fashion. ${ }^{1}$ The World Health Organization (WHO) on January 30, 2020, confirmed the extensive increase in COVID-19 cases a public health crisis. Thus, there has to be a paradigm shift in the way healthcare procedures are done across the world in accordance with the guidelines set by $\mathrm{WHO}^{2,3}$

Dentistry is unique in the sense that not only the operator and the patient are in close immediacy during the examination and the procedure but also the main source of infection is the actual area of operation - the oral cavity. In dental settings, oral fluids like blood and saliva from the patient or infected dental instruments can create a possible way of distribution of the virus to the dentist, assistant, and to the patients. Customary dental procedures that include the use of rotating instruments such as the high-speed turbine handpiece and the use of ultrasonic scalers for oral prophylaxis are allied with the production of large quantity of aerosols and droplets from the saliva and blood of the patient. Before settling on the environmental surfaces and on the medical instruments, these can remain suspended in the air for a long period and can penetrate the respiratory tract through the nose and mouth. ${ }^{4}$ Hence, it is apparent how the aerosols generated during dental treatment can render the operators and patients to the menace of cross-infection. This review aims to discuss alternate ways in which the pediatric dental interventions can be done, which can be a great way of restoring the pediatric patient back to health. These options are aerosol-free and thus can contain the contagion.

Dental management of pediatric patients in the period of pandemic should be based on the severity of the case, the degree of invasiveness of the procedure, and the risk involved.

\section{Initial Evaluation and Preventive Dental CARE}

For initial patient screening and evaluation of the problem, teledentistry is the best method. ${ }^{5}$ A detailed travel, medical, and dental history should be taken over phone to understand the problem and needs of the patient. This shall help in reduced patient $\overline{\text { 1,2 Department of Dentistry, Shaheed Hasan Khan Mewati Government }^{2}}$ Medical College, Nuh, Haryana, India

Corresponding Author: Megha B Jain, Department of Dentistry, Shaheed Hasan Khan Mewati Government Medical College, Nuh, Haryana, India, Phone: +91 7032909017, e-mail: mb30in@yahoo.co.in

How to cite this article: Sharma A, Jain MB. Pediatric Dentistry during Coronavirus Disease-2019 Pandemic: A Paradigm Shift in Treatment Options. Int J Clin Pediatr Dent 2020;13(4):412-415.

Source of support: Nil

Conflict of interest: None

and dentist contact time and proper appointment scheduling Pediatric dentist should use various "social" digital platforms on which they can circulate and propagate behavioral guide for the safety of the dental health of children. Child patients requiring regular checkups and follow-ups with no eventful history should be completely deferred for some time.

Preventive care instructions like (1) brushing twice daily with fluoridated toothpaste, (2) minimal consumption of sweet and sticky food, (3) rinsing after every meal, and (4) more of fruits and healthy food in the diet can be explained over the phone or social media applications.

\section{Dental Management of Cavitated Teeth without Any Pulp Involvement}

Dental management of cases requiring restorative care should be carefully evaluated taking a proper history and clinical examination. The cavitated tooth not involving the pulp can be treated by following methods:

\section{Atraumatic Restorative Technique}

Atraumatic restorative technique (ART) is a time-tested technique making restorative care accessible for pediatric patients and has high success rates. ${ }^{6}$ This technique is highly advantageous for young children who are fearful to the conventional drill and local anesthesia. It has found special relevance in times of the pandemic as it promotes no aerosol dentistry. The main indications are that it 
can be carried out only in small and shallow cavities. The technique involves the scooping out of spongy, demineralized tooth tissue using manual instruments alone (spoon excavator) and then filling the cavity with adhesive curative material, generally glass ionomer cement $(\mathrm{GIC})^{7}$

\section{Silver Diamine Fluoride}

Silver diamine fluoride can arrest dental caries and prevents its progression. It was originally used in Japan in 1970s but soon fell out of favor due to the unaesthetic discoloration. Now, many countries have recommended the use of $38 \%$ silver diamine fluoride (SDF) solution for caries prevention as well as for caries arrest. ${ }^{8}$ Silver diamine fluoride is a colorless liquid that combine the remineralizing property of fluoride and the antibacterial effects of silver. For managing carious lesions in young children and those with special care needs, it is therefore a potentially capable remedial agent. ${ }^{9}$

The oral cavity is inspected for cavitated lesion and it is advisable to probe the lesion to check for the extent. Silver diamine fluoride should not be used in case the carious lesion is approaching the pulp or is found to be too deep. The carious lesion is completely dried using cotton rolls and the solution is applied over it using an applicator tip. The application time is 1 minute. A clear-cut discoloration of the carious lesion to black shall be seen, signaling the end of application. For cavitated lesions on coronal or root surfaces that are not suspected to have pulp involvement, are not symptomatic, and are cleansable, SDF is indicated. ${ }^{9}$

It is suggested that SDF primarily acts by three mechanisms. First, it may cause the obturation of dentinal tubules. The second mechanism is the cariostatic action of the resultant products between SDF and mineral constituent of the tooth. The third proposed way is the anti-enzymatic actions of the reaction yield between $\mathrm{Ag}\left(\mathrm{NH}_{3}\right)_{2} \mathrm{~F}$ and organic component of the tooth. ${ }^{8}$

The biggest advantage with SDF is that there is no cavity preparation; hence, it eliminates the usage of airotors/micromotors completely. There is control of pain, infection, and minimal armamentarium is needed. The biggest disadvantage is the obvious discoloration of the tooth to black. However, this can be overcome to some extent by placing layer of GIC over the cavitated lesion. ${ }^{9}$

\section{Hall Crown Technique}

The Hall crown technique using preformed metal crowns was introduced by Dr Norna Hall from Scotland in 2006. ${ }^{10}$ They are stainless steel crowns that are used in the cavitated lesions. Just like SDF, there is no cavity preparation involved here. Indication involves class I or class II cavitated or noncavitated primary molar tooth. Orthodontic separators are put in the place for 3-7 days on the proximal sides of the tooth to be restored. Once optimal space is created, the crown is cemented into place using a luting agent. ${ }^{11}$ The crown gives full veneering advantage and prevents the progression of caries to the pulp as well as to the neighboring tooth. The patient is thereafter kept on a regular follow-up.

\section{Chemomechanical Caries Removal}

It is a newer noninvasive technique of removing carious infected dentin via a chemical agent. It is of caries elimination based on dissolution. Instead of drill, this method uses a chemical agent along with an atraumatic mechanical force to get rid of soft carious structure. It complies with the theory of the minimal invasive dentistry (MID). ${ }^{12}$

The procedure involves applying of solution/gel to the carious dentin, allowing it to soften the tissue, and thereafter scraping it off with specialized hand instruments. The two most currently sought agents are Cariosolv and Papacarie.

Carisolv consists of:

- Syringe 1: Sodium hypochlorite (0.5\%)

- Syringe 2: Pink viscous gel having three amino acids: leucine, glycine, and lysine.

When the components of two syringes are mixed as one, the amino acids bind with chlorine and form chloramines. This results in the breakdown of degraded collagen found in the demineralized part of carious lesion. The gel softens only carious tissue, which is then removed with special hand instruments. ${ }^{12}$

Papacarie consists of papain enzyme (which is an extract of the fruits and the latex of leaves of the green adult Carioca papaya tree), Toluidine blue, chloramine, a thickener, salts, stabilizers, preservatives, and deionized water. The mechanism of action depends on the papain enzyme, which is a proteolytic enzyme that causes degradation of proteoglycans in the dentinal matrix. It has bactericidal and anti-inflammatory actions. Chloramine enhances the removal of denatured tissues. ${ }^{13}$

\section{Dental Management of Cavitated Tooth With Pulp Involvement}

\section{Reversible Pulpitis}

In a deciduous tooth with reversible pulpitis lacking evidence of radicular pathology, the pulpotomy procedure can be performed. By use of low-speed micromotor handpiece, access can gained be into coronal pulp. The coronal pulp is removed while the residual vital radicular pulp tissue is maintained and the surface is treated with a clinically proven medicament such as Buckley's Solution of formocresol. ${ }^{14}$ Mineral trioxide aggregate (MTA) and calcium hydroxide are materials used for pulpotomies with an elevated rate of success. ${ }^{15}$

In young permanent teeth with reversible pulpitis, partial or Cvek's pulpotomy can be done. It is a procedure in which the inflamed pulp tissue underlying an exposure is removed to a depth of 1-3 $\mathrm{mL}$ or further to reach the healthy tissue. Pulpal hemorrhage is controlled using bactericidal irrigants such as chlorhexidine or sodium hypochlorite thereafter which the site is covered with calcium hydroxide or MTA. It is preferred in teeth with immature roots to continue apexogenesis and usual root development. $^{14}$

\section{Irreversible Pulpitis}

Pulpectomies in primary teeth should be avoided as it might require multiple sittings depending on the clinical condition of the tooth and extensive instrumentation during biomechanical preparation shall increase patient and dentist's contact time. Therefore, extraction of the affected tooth is recommended in such a case. Extraction followed by space maintainers would ensure prevention of adjoining tooth moving in to occupy the space. ${ }^{16}$

In young immature teeth, full or cervical pulpotomy can be done unless the pulp is found to be necrotic after entering the pulp chamber. The remaining pulp will heal as long as hemorrhage is controlled. Thereafter, biocompatible materials like calcium hydroxide or MTA are applied. When pulpotomies are performed in young permanent teeth, the tooth may require reentry in future for root canal treatment, if signs and symptoms of pulpal necrosis appear. $^{14}$ 


\section{Dental Management of Emergency Situations}

\section{Extraoral Swelling}

In case the swelling is related to a carious tooth indicated for extraction, extraction under proper antibiotic coverage should be the treatment of choice. If the swelling involves facial spaces then incision and drainage along with proper antibiotic coverage and pain management should be the mode of treatment. ${ }^{5}$

\section{Dental Trauma}

In case of soft tissue injury, the patient should be first evaluated over telephone by taking a detailed history and assessing the site of lesion through the photographs via digital applications. Parents should be advised to control the bleeding through pressure pack or cold compression. In case bleeding does not stop, the patient should be asked to report to the dental operatory so that suitable management can be done.

In case of mobile deciduous tooth, splinting should be done using self-etch composite resin material with minimal postprocedure finishing. In case of avulsion of primary tooth, control of bleeding should be the main mode of treatment followed by pain management of the child.

If case of avulsed permanent tooth, the patient should be directed to get the tooth immediately to the dental clinic wherein the tooth should be reimplanted followed by splinting the tooth to maintain the repositioned tooth in correct position, provide comfort to the patient, and maintain function. Other facial traumatic injuries should be attended on emergency basis and guidelines of the International Association of Dental Traumatology (IADT) ${ }^{17,18}$ should be followed as far as possible.

\section{Dental Management of Orthodontic EMERGENCIES}

\section{Removable Appliances}

These include functional appliances and retainers. Growing children are given functional appliances to correct the growth of jaws and the dentoalveolar complex. If the patients has noticeable discomfort wearing the appliance or breaks it, the use of the appliance should be temporarily suspended. A retainer appliance can be frequently lost by the patient or broken down; in such a case, it is advised that the clinicians take new impressions or digital scans and remake the retainer. ${ }^{19}$

\section{Fixed Appliances}

A bracket may lose its metallic or elastic ligature or become loose as a result of eating sticky or hard food. If the bracket remains flush with the tooth, it can be left as it is, if it seems to drop from the archwire, the patient can cautiously try to remove it with eyebrow tweezers. If there is a metallic ligature that causes soft tissue pain or injury, the patient should aim to push it back with the small eraser on the back of a pencil. In case that it is not possible, then orthodontic relief wax can be applied. ${ }^{19}$

\section{General Instructions to be Followed in the Dental Operatory}

The COVID-19 pandemic presents unique challenges to dentistry. With newer ways of working and added stress on disinfection, the fraternity has to adapt quickly. Following are general precautionary measures to be followed at all dental operatories:

- Mandatory use of rubber dam in all procedures. ${ }^{20}$

- Using $0.23 \%$ povidone-iodine mouthwash for at least 15 seconds or $0.5-1 \%$ hydrogen peroxide mouth rinse before the procedure is recommended as it can reduce the viral load in the patient's saliva. $^{21}$

- Extraoral dental radiographies, such as panoramic radiography and cone-beam CT, are suitable alternatives through the spread of COVID-19 pandemic. $^{5}$

- Personal protective equipment kits involving gown, goggles, face shield, etc., to be worn by the dentist and assistant at all times during the procedure. ${ }^{5}$

- Use of throwaway (single-use) devices such as mouth mirror and syringes to avert cross-contamination is adviced. ${ }^{5}$

\section{Conclusion}

Dental professionals have the responsibility to guard the patient, auxiliary staff, and themselves and also uphold high standards of care and infection control while delivering the treatment. With multiple options present to deal with the different clinical scenarios, it is up to the dentist to make a judicious call.

\section{References}

1. Centers for Disease Control and Prevention. Transmission of Coronavirus disease 2019 (COVID-19). https://www.cdc.gov/ coronavirus/2019-ncov/about/transmission.html.

2. WHO, Critical preparedness, readiness and response actions for COVID-19. Available at https://www.who.int/publicationsdetail/ critical-preparedness-readiness-and-response-actionsfor-covid-19.

3. Centers for Disease Control and Prevention. Recommendations for putting on and removing personal protective equipment. https:// www.cdc.gov/hai/pdfs/ppe/ppe-sequence.pdf.

4. Kampf G, Todt D, Pfaender S, et al. Persistence of corona viruses on inanimate surfaces and their inactivation with biocidal agents. J Hosp Infect 2020;104(3):246-251. DOI: doi.org/10.1016/j.jhin.2020.01.022.

5. Ather A, Patel B, Ruparel NB, et al. Coronavirus disease 19 (COVID-19): implications for clinical dental care. J Endod 2020;46(5):584-595. DOI: 10.1016/j.joen.2020.03.008.

6. Saber AM, El-Housseiny AA, Alamoudi NM. Atraumatic restorative treatment and interim therapeutic restoration: a review of literature. Dent J 2019;7(1):28-38. DOI: 10.3390/dj7010028.

7. Carvalho T-S, Ribeiro T-R, Bönecker M, et al. The atraumatic restorative treatment approach: an "atraumatic" alternative. Med Oral Patol Oral Cir Bucal 2009;1(14):668-673. DOI: 10.4317/medoral.14.e668.

8. Shah S, Bhaskar V, Venkatraghavan K, et al. Silver diamine fluoride: a review and current applications. J Adv Oral Res 2014;15(1):25-35. DOI: $10.1177 / 2229411220140106$.

9. Galui S, Pal S, Pabale SL, et al. Stretching new boundaries of caries prevention with silver diamine fluoride: a review of literature. Int J Pedod Rehabil 2018;3(1):1-4.DOI: 10.4103/ijpr.ijpr_32_17.http://www. ijpedor.org/text.asp?2018/3/1/1/227953.

10. Altoukhi $\mathrm{DH}, \mathrm{El}-\mathrm{Housseiny} \mathrm{AA}$. Hall technique for carious primary molars: a review of the literature. Dent J 2020;8(1):1-10. DOI: 10.3390/ dj8010011.

11. Batool $G$, lyad $H$. The Hall technique in paediatric dentistry: a review of the literature and an "all Hall" case report with a-24 month follow up. Stoma Edu J 2017;4(3):208-217. DOI: 10.25241/stomaeduj.2017.4 (3).art.6.

12. Ganesh M, Dhaval. P. Chemomechanical caries removal (CMCR) agents: review and clinical application in primary teeth. J Dent Oral Hyg 2011;3(3):34-45. http://www.academicjournals.org/JDOH. 
13. Mithra NH, Abhishek M. Chemomechanical caries removal: a conservative and pain-free approach. Adv Res Gastroentero Hepatol 2017;5(3):69-71. DOI: 10.19080/ARGH.2017.05.555666.

14. American academy of pediatric dentistry. Pulp Therapy for primary and immature permanent teeth. The reference manual of pediatric dentistry. 2019-2020: pp. 353-361.

15. Mortazavi M, Mesbahi M, Azar MR, et al. MTA pulpotomy in primary molars: a prospective study. Res J Biol Sci 2009;4(5):1-5.

16. Wilson B, Joseph J, Bharadwaj $P$, et al. Space management in paediatric dentistry. J Den Pana 2014;1(2):68-76. DOI: 10.15636/ jdp/2014/v1i2/5842.

17. Diangelis AJ, Andreasen JO, Ebeleseder KA. Guidelines for the management of traumatic dental injuries: 1 . fractures and luxations of permanent teeth. Pediatr Dent 2017;39(1):401-411. DOI: 10.1111/j.1600-9657.2011.01103.x.
18. Andersson L, Andreasen JO, Day P, et al. Guidelines for the management of traumatic dental injuries: 2 . avulsion of permanent teeth. Pediatr Dent 2017;39(2):412-419. DOI: 10.1111/j.16009657.2012.01125.x.

19. Caprioglio A, Pizzetti GB, Zecca PA, et al. Management of orthodontic emergencies during 2019-NCOV. Prog Orthod 2020;21(10):1-4. DOI: 10.1186/s40510-020-00310-y.

20. Cochran MA, Miller $\mathrm{CH}$, Sheldrake MAT. The efficacy of the rubber dam as a barrier to the spread of microorganisms during dental treatment. J Am Dent Assoc 1989;119(1):141-144. DOI: 10.14219/jada. archive.1989.0131.

21. Eggers $M$, Koburger-Janssen T, Eickmann $M$, et al. In vitro bactericidal and virucidal efficacy of povidone-iodine gargle/mouthwash against respiratory and oral tract pathogens. Infect Dis Ther 2018;7(2):249259. DOI: 10.1007/s40121-018-0200-7. 